\title{
Frontières
}

\section{Peur et tremblements}

Autour de Hard Candy et The Brave One

\section{Martine Delvaux}

Volume 21, numéro 2, printemps 2009

Détresse psychique et antidépresseurs

URI : https://id.erudit.org/iderudit/039454ar

DOI : https://doi.org/10.7202/039454ar

Aller au sommaire du numéro

\section{Éditeur(s)}

Université du Québec à Montréal

ISSN

1180-3479 (imprimé)

1916-0976 (numérique)

Découvrir la revue

Citer cet article

Delvaux, M. (2009). Peur et tremblements : autour de Hard Candy et The Brave One. Frontières, 21(2), 27-31. https://doi.org/10.7202/039454ar
Résumé de l'article

Que se passe-t-il lorsqu'une femme préfère la rage à l'anesthésie des comprimés ? C'est ce qu'ont fait les personnages féminins de Hard Candy et The Brave One, en se faisant vigilante pour se faire justice. Par une lecture de l'intertexte du Petit Chaperon rouge, l'article montre que la victime et la bête ne sont parfois qu'une même personne et que si la jeune fille entre dans la gueule du loup, c'est seulement pour mieux le tailler en pièces. d'utilisation que vous pouvez consulter en ligne.

https://apropos.erudit.org/fr/usagers/politique-dutilisation/ 


\section{Résumé}

Que se passe-t-il lorsqu'une femme préfère la rage à l'anesthésie des comprimés? C'est ce qu'ont fait les personnages féminins de Hard Candy et The Brave One, en se faisant vigilante pour se faire justice. Par une lecture de l'intertexte du Petit Chaperon rouge, l'article montre que la victime et la bête ne sont parfois qu'une même personne et que si la jeune fille entre dans la gueule du loup, c'est seulement pour mieux le tailler en pièces.

Mots clés: Chaperon rouge - loup vengeance - victime - vigilantes.

\section{Abstract}

What happens when a woman chooses rage over anti-depressants? This is what the female protagonists of Hard Candy and The Brave One have done, becoming vigilantes in order to do justice to themselves and other women. Through a reading of the movies' little red riding hood intertext, this article shows how the beast and the victim are sometimes one and the same, and if a girl lets herself eaten up by a wolf, it is to better turn the tables on him.

Keywords: Red Riding Hood - wolfvengeance - victim - vigilantes.

\title{
PEUR ET TREMBLEMENTS Autour de Hard Candy et The Brave One
}

\author{
ON VOIT ICI QUE DE JEUNES ENFANTS, \\ SURTOUT DE JEUNES FILLES, \\ BELLES, BIEN FAITES ET GENTILLES \\ FONT TRÈS MAL D'ÉCOUTER TOUTES SORTES DE GENS, \\ ET QUE CE N'EST PAS CHOSE ÉTRANGE, \\ S'IL EN EST TANT QUE LE LOUP MANGE. \\ JE DIS LE LOUP, CAR TOUS LES LOUPS \\ NE SONT PAS DE LA MÊME SORTE: \\ IL EN EST D'UNE HUMEUR ACCORTE, \\ SANS BRUIT, SANS FIEL ET SANS COURROUX, \\ QUI, PRIVÉS, COMPLAISANTS ET DOUX, \\ SUIVENT LES JEUNES DEMOISELLES \\ JUSQUE DANS LES MAISONS, JUSQUE DANS LES RUELLES. \\ MAIS, HÉLAS! QUI NE SAIT QUE CES LOUPS DOUCEREUX, \\ DE TOUS LES LOUPS SONT LES PLUS DANGEREUX!
}

\author{
CHARLES PERRAULT
}

\author{
Martine Delvaux, Ph.D, \\ professeure titulaire, Département \\ d'études littéraires, UQAM.
}

À la fin de son ouvrage sur le petit chaperon rouge Red Riding Hood Uncloaked (2003), Catherine Orenstein décrit une des poupées de son enfance, poupée réversible et multiface qui, selon le désir de l'enfant, pouvait représenter les différents personnages d'un conte de fées. Les personnages pouvaient apparaître un à la fois, jamais tous en même temps. Pour Orenstein, cette figure décrit la structure du conte de Perrault, Le Petit Chaperon rouge: la petite fille, la grand-mère et le loup sont les différents alias d'un même corps, qui coexistent sans jamais pouvoir être présents au même moment. Cette poupée rappelle la parallaxe explorée par Slavoj Zizek dans The Parallax View (2006), la modification d'un objet observé en fonction du déplacement du point de vue de l'observateur. Les perspectives sont irréconciliables, contradictoires ${ }^{1}$, ce qui ne signifie pas qu'elles sont conflictuelles mais que cette différence radicale entre les façons de «voir les choses» pointent à la tension, à la non-coïncidence de la réalité avec ellemême. Suivant la parallaxe, on ne pourrait 
pas saisir le réel pour ce qu'il est, on ne pourrait jamais voir les choses telles qu'elles sont. Dans les pages qui suivent, je veux me pencher sur deux films récents, Hard Candy (2005) et The Brave One (2007), qui, dans ce qui semble être une relecture du Petit Chaperon rouge, mettent en scène des vigilantes féminins - une fille et une femme qui deviennent meurtrières à la suite de violences exercées sur leur propre corps et celui de proches. Ces femmes sont d'emblée présentées comme souffrantes mentalement (de façon ironique dans Hard Candy, de manière littérale dans The Brave One), mais la détresse psychique, au lieu d'être abolie par le traitement devient le moteur d'une rage vengeresse - la colère, au lieu d'être calmée, est exploitée.

« 4 out of 5 doctors agree that I am insane», annonce le personnage d'Ellen Page, jeune adolescente de 14 ans, à Jeff Kohlver, le pédophile avec qui elle a entretenu une relation virtuelle via Internet et qu'elle sait responsable de la disparition d'une adolescente, Donna Mauer. Plus tard, quand le loup-pédophile se réveillera entre les mains de la jeune fille, drogué puis attaché par celle qui se prépare à le castrer (pour l'amener éventuellement à se suicider), il crie: "You need help! $A$ teenager doesn't do this!... I'll pay for a therapist. » Le scénario de Hard Candy est ponctué de renvois à la santé mentale de Haley Stark, soit dans sa bouche à elle qui assume volontiers ce qu'elle appelle non sans ironie sa folie, soit dans la bouche de Jeff qui tente d'obtenir sa compassion en tentant de faire usage de psychologie. D'une façon analogue, l'héroïne de The Brave One, Erica Bain - dont on suit le déclin d'animatrice radio fiancée heureuse et nonchalante à survivante effondrée et terrorisée, en passant par la vision de son corps violenté et comateux - jette les comprimés qu'on lui a prescrits au sortir de l'hôpital pour combattre son stress posttraumatique. Elle remplace ce palliatif par une arme achetée illégalement, qu'elle utilise pour mettre à mort des criminels dont les cibles de prédilection sont des femmes ${ }^{2}$.

Dans la lignée du Travis Bickle de Taxi Driver (Scorcese, 1999 [1976]), Haley Stark (Ellen Page) et Erica Bain (Jodie Foster), les héroïnes de Hard Candy et The Brave One ${ }^{3}$, d'une part, incarnent une certaine précarité psychique, d'autre part, prennent en main la justice, vengeant filles et femmes en réglant leur compte à des pédophiles, proxénètes, voyous, violeurs, et assassins. Là où Travis Bickle était fait de pulsions déchaînées par une mésadaptation et ce que le scénario nous présente comme une forme de folie (restes de l'expérience militaire au Viêt-nam), Haley Stark et Erica Bain apparaissent comme des êtres pourvus d'une conscience et d'un sens moral, qui désobéissent au murmure social qui dit que les filles, les femmes sont essentiellement des victimes. Elles refusent de consentir, d'apprendre la leçon de souffrance et de terreur qui semble leur avoir été dictée. La détresse n'est pas ici la cause d'une anesthésie (médicamentée ou non); elle est celle d'une action qui, au-delà de la survie de la femme qui la commet, a pour objectif de protéger et de venger d'autres comme elle. Le neighborhood watch est autre chose que de la surveillance passive, c'est un véritable passage à l'acte. Haley Stark et Erica Bain traquent et punissent les criminels comme dans une version du Petit Chaperon rouge où c'est le chaperon qui prend au piège le loup dans le but d'empêcher d'autres attaques sur d'autres petites filles comme elle.

Les contes de fées le disent: les petites filles doivent être douces et gentilles, passives, soumises, muettes, vouées à l'attente et, surtout, elles doivent obéir à leurs parents. N'est-ce pas ce qu'écrit Charles Perrault à la fin du Petit chaperon rouge? Les jeunes filles «belles, bien faites et gentilles font très mal d'écouter toutes sortes de gens ». Les filles sont des filles de père, du père-justice, du père-loi, et entre lui et le pire (Zizek, 2006), c'est toujours lui qu'elles doivent choisir. Mais si, comme le propose Orenstein (2003), le petit chaperon rouge et le loup étaient deux aspects d'un même sujet? Disons qu'on opère une parallaxe et que le petit chaperon rouge et le loup apparaissent comme deux perspectives différentes et irréconciliables (mais indémaillables) sur le même objet. Et si cette petite fille douce et naïve était le loup? Et si, au lieu de se complaire dans son rôle de petite fille soumise, elle choisissait d'occuper la place du loup, de se laisser dévorer par lui pour l'habiter, prendre sa peau? Si elle choisissait l'inconfort de la parallaxe, habiter la zone de tension, plutôt que la passivité anesthésiée? Elle serait alors à la fois la belle et la bête. Elle ne serait plus Peau d'Âne mais Peau de Loup, et qu'est-ce que ça voudrait dire pour le pouvoir (la colère, la rage, la vengeance) des femmes?

Si la presse et la culture populaire (talkshows, séries télévisées, magazines féminins...) ont tendance à présenter les mères comme des êtres dépressifs, emprisonnés dans un infini post-partum, Hard Candy et The Brave One ouvrent la porte sur une autre mère, une fille-mère ${ }^{4}$ qui ne tait pas sa détresse avec des comprimés et qui agit sur sa colère. C'est elle qui fait la leçon - pensons à l'importance des monologues des héroïnes dans les deux films - et de sa vigilance, l'outil d'une réparation. Elle nomme ce qu'on veut taire, condamne ceux qu'on ignore ou qu'on protège. Elle est une femme en colère, une mère qui peut se faire chaperon rouge pour attirer le loup, une mère-loup qui protège à tout prix le chaperon rouge, une femme qui, entre le père-justice et le père-loup choisit le second.

Hard Candy et The Brave One font bien résonner le conte de Perrault, Hard Candy de façon délibérée (l'affiche propose clairement Le petit chaperon rouge comme intertexte), The Brave One moins frontalement. On trouve donc nombre d'échos: la veste rouge à capuchon portée par Haley Stark au début du film qu'elle retire dès le début pour ne la remettre qu'à la toute fin, une fois le pédophile suicidé ; le chienloup d'Erica Bain, utilisé par ses agresseurs pour l'attirer ainsi que son fiancé dans leur piège (animal qui sera libéré à la fin du film, une fois l'ultime vengeance accomplie contre les agresseurs); le travestissement par les protagonistes qui troquent rapidement la jupe pour le pantalon (et dans le cas de Haley Stark, pour le pyjama de chirurgien) ; la visite de Haley Stark à la maison du pédophile qui semble presque isolée au sein de la ville et dont la forme carrée est sans cesse accentuée (le cadrage de la caméra enchâssant des murs sur lesquels sont fixés des tableaux), figurant à l'infini l'emboîtement qui est au cœur du conte de Perrault ${ }^{5}$, comme si l'écran constituait les pages d'un livre aux couleurs d'un conte pour enfants; enfin, la déambulation d'Erica Bain dans les rues de New York, la nuit, comme le petit chaperon rouge dans la forêt... Autant de topoi qui évoquent la forme du conte et le contenu qu'il traduit.

De fait, si voir le loup, c'est perdre sa virginité, Hard Candy et The Brave One racontent tous deux la perte de l'innocence due à l'expérience de la violence : la disparition d'une amie et une conscience acerbe de la pédophilie chez Haley Stark; la survie de Erica Bain à une attaque au cours de laquelle son fiancé a perdu la vie. Et comment continuer à vivre quand on a vu le loup? Comment une fille, une femme peut-elle «trouver le moyen de continuer à vivre» (pour reprendre les mots de la voisine de Erica Bain dans The Brave One) dès qu'elle cesse d'être ce petit chaperon rouge "d'avant l'information, d'avant la connaissance, d'avant le langage qui organisent l'ordre et le désordre du monde»(Garat, 2004, p. 58) ? Ni auteure, ni lectrice, le petit chaperon rouge est non seulement sans imagination (elle ne peut prévoir les risques) mais incapable de sémiologie. Haley Stark et Erica Bain, elles, deviennent des vigilantes. Elles apprennent à décoder les signes, à lire le monde et à s'y inscrire comme sur un 
échiquier. Circulant dans New York après son agression, Erica Bain se demande si c'est elle qui trouve de nouveaux lieux, ou si ce sont ces lieux qui la trouvent elle ${ }^{6}$. Après avoir été initiées à la violence, Haley Stark et Erica Bain ont les yeux grands ouverts, décillés. Si elles dansent avec le loup, c'est pour le tuer, rendant la monnaie de sa pièce à celui qui dévore les fillettes en une seule bouchée ${ }^{7}$.

Dans les deux scénarios, une même menace est proférée par un homme à leur endroit: "Ever been fucked by a knife?" (The Brave One) / "Which do you want to fuck first, me or the knife?" (Hard Candy). Ordinateur (traitement de texte, chat et courriel), scalpel (techniques médicales), fusil (techniques policières), téléphone cellulaire (moyen de communication)... entre les mains de ces femmes, le dispositif est détourné, tourné contre celui qui a l'habitude de le posséder:

I gotta wonder about a grown man who goes to such trouble to charm a girl. Hey! There's that word again! GIRL. Maybe it's the camera thing. Computers, cameras, they let you hide a little, don't they? So safe. I heard how your voice changed when you got this [camera] between us (Haley Stark à Jeff).

La femme ordinaire est bien une terroriste, retournant contre le loup-système les objets qu'il a créés pour la dominer, laissant le dispositif à l'usage du monstre qui se trouve à l'intérieur d'elles. Contrairement au petit chaperon rouge avalé par le loup (et qu'un chasseur libère dans la version des frères Grimm $^{8}$ ), ici, c'est le petit chaperon rouge qui a avalé le loup, qui l'a peut-être même toujours porté à l'intérieur d'elle.

Dans sa lecture du Petit chaperon rouge, Anne-Marie Garat décrit le loup comme:

[...] terré au coin de notre Forêt mentale, loin du Village utopique des hommes, tout près de nous, enfouis sous nos draps, il approche sa peau de notre peau, frotte son poil à notre poil, vieille connaissance. Vieille terreur de connaissance. Vieux compère de nos nuits. [...] La beauté paradoxale du conte, sa séduction atroce, c'est que l'apparition du Loup de Perrault, pour être subreptice et violente, en cela conforme à la tradition, ne surprend pas une seconde.

Ni Chaperon rouge, ni nous, lecteurs, qui sommes d'intelligence avec cette histoire très vieille de rencontre et de dévoration, familiers de nos monstres intérieurs (Garat, 2004, p. 68-69).

Pour Peter Sloterdijk, les temps modernes sont l'ère du monstrueux créé par l'homme. «Est moderne, écrit-il, celui qui est touché par la conscience du fait que lui ou elle, au-delà de l'inévitable qualité de témoin, est intégré par une sorte de complicité à ce monstrueux d'un nouveau type» (Finkielkraut et Sloterdijk, 2003, p. 205). Le moderne, selon Finkielkraut et Sloterdijk, à l'heure du crime, se trouve sur le lieu du crime. Il n'est pas un innocent bystander, il n'a plus d'alibi, il participe de cet excès, se trouve là où le «beaucoupplus est provoqué, commis et attesté [...] On y a part comme à une fièvre qui incite sa victime à trouver un nouveau mode d'être, au-delà de la santé et de la maladie » (Finkielkraut et Sloterdijk, 2003, p. 206). N'est-ce pas ce nouveau mode d'être que découvre Erica Bain après son agression, ce «monstre» qui se révèle à l'intérieur d'elle, cet étranger? Comme un organisme qui mute pour s'adapter à son nouvel environnement ${ }^{9}$ afin de ne pas disparaître, c'est ce monstre, sorte d'excroissance, excès produit en réaction à l'agression, qui désormais la fait agir, parler.

The Brave One s'ouvre sur cette speakerine dont la voix (et non le visage: «I'm not a face, I'm just a voice») traverse New York par le biais des ondes radiophoniques: "I'm Erica Bain and I walk the city. » Après avoir quitté l'hôpital et tenté de reprendre une vie normale, elle est témoin d'un meurtre - un soir, alors qu'elle achète du lait dans un dépanneur, elle voit un homme assassiner la caissière, sa femme, parce qu'elle lui refuse l'accès aux enfants. Lorsque l'homme se rend compte de la présence d'Erica Bain dans le magasin, il se met à sa poursuite. Le duel prend fin sur sa mort. À la suite de cet événement, décrivant la transformation d'un individu par la peur et la nouvelle perception de la ville qui s'ensuit, Erica Bain dit sur les ondes: "Inside you there is stranger, sleepless, restless stranger, who keeps walking, eating, living. » Plus tard, quand le policier / ami lui demande comment elle a fait pour se remettre de ce qui lui est arrivé, elle répond: "You don't... you become someone else. A stranger. » $\mathrm{Si}$ cet étranger est le visage de la peur - celle des femmes qui rentrent seules chez elles, ou de la poudre blanche dans la boîte postale... - il devient bientôt le visage de la vengeance.

Erica Bain ne se reconnaît plus, ses gestes lui sont étrangers comme l'est aussi, après le crime, son absence de réaction ("Why don't my hands shake?») et celle des autres, d'un autre, abstrait ou singulier qui pourrait l'arrêter («Why doesn't somebody stop me?»). À la manière du loup habillé en mère-grand, Erica Bain se travestit - «Hey you» dit-elle froidement devant son image dans le miroir.
On la dirait en uniforme, entre flic et soldat, revêtant pantalon et t-shirts sombres décorés sur le devant, quelque part entre homme et femme, bourreau et victime. Pour la trouver, les policiers sont forcés de penser "outside of the box», d'aller contre la logique habituelle ("Women kill shit they love, they don't do this»). Ils se mettent bientôt à chercher " a woman with a grudge», cette femme possédée par un étranger, qui n'est plus rien d'autre que lui : "There is no going back. This thing, this stranger, this is all you are now."

La transformation de Erica Bain pourrait se résumer au passage de la première à la deuxième personne. Soudain, elle devient les autres, se trouve redoublée par l'autre - à la fois le parfait étranger et madame tout le monde. C'est la parallaxe du you, ce pronom qui peut nommer soi et l'autre. You est un moteur de subjectivation et de désubjectivation qui met en langage la précarité de l'identité; c'est un mouvement de bascule, une hésitation entre le désir de prendre à son compte et celui de déposer chez l'autre ce qui renvoie à soi. Le you est une sorte de citation, la marque grammaticale, dans ce cas-ci, du geste de désobéissance, du crime. Mais si c'est le nom de l'étranger (le nom de cet alien qu'Anne-Marie Garat voit dans le loup travesti en grand-mère dans le conte de Perrault), quel est le sens de cette figure de l'étrangeté pure? Au lieu d'être perçue comme une aliénée, rendue étrangère à elle-même par la force des "dispositifs», cette société de "corps inertes travers[ée] par de gigantesques processus de désubjectivation auxquels ne répond aucune subjectivation réelle»(Agamben, 2007, p. 46), Erica Bain suit les mouvements de l'autre en elle comme elle marche dans New York (ville étrangère à elle-même depuis les attaques du 11 septembre) traquant les monstres extérieurs - les truands - qui menacent son espèce. L'alien n'est pas un alibi ; il est le moyen de se tailler une autre place, se retrouver ailleurs ${ }^{10}$.

De façon analogue, le personnage de Haley Stark se confond avec un groupe non identifié de petites filles, des filles sans visage, toutes victimes, avérées ou potentielles, de la pédophilie: "I'm every little girl you ever watched. Touched. Hurt. Screwed. Killed. And we're all back now to cut you off. " Usant elle aussi des dispositifs, Haley Stark joue le jeu de l'anonymat, de la petite fille ordinaire tombée dans le piège du méchant loup, pour faire apparaître sur son visage les fantômes de celles qui sont disparues ou qui sont en danger. Comme si les filles, les femmes étaient une espèce en voie de disparition qu'il fallait protéger, combattre les prédateurs et les chasseurs, les vautours ${ }^{11}$. 
LA FEMME-MONSTRE, LA FEMME-LOUP EST CE QUE DEVIENT LE PETIT

CHAPERON ROUGE, NON PAS PARCE QU'IL N'A PAS BIEN APPRIS

\section{SA LEÇON MAIS PARCE QUE LA LEÇON ÉTAIT UN LEURRE, UNE FAÇON}

DE PRÉPARER LA VICTIME. PAR LES CRIMES QU'ELLES COMMETTENT,

LES FEMMES DE HARD CANDY ET THE BRAVE ONE EXPRIMENT

LEUR ÉTAT DE TÉMOIN MODERNE, DE TÉMOIN TOUJOURS COMPLICE

\section{DE LA MODERNITÉ ET DE SES DISPOSITIFS.}

Comme si ce qui arrive aux femmes, ce qu'on leur fait, représentait un dérapage analogue aux effets du "progrès » technologique sur l'environnement naturel: que peut devenir une fille, une femme quand elle vit sous la menace constante? Au lieu de sombrer dans la dépression, elle peut en venir à révéler une seconde nature. Elle peut muter, se faire "monstre» grâce à l'effet de la peur, quand la peur devient ce dont on ne peut pas revenir, quand on sait que ce qu'on craint de perdre a déjà été perdu (Zizek, 2006, p. 434). C'est alors que la peur bascule du côté de la terreur, le fait de trembler devient l'opposition la plus radicale à la peur (plutôt que son synonyme ou son symptôme), et au lieu de chercher à se protéger, on oublie l'objet parce qu'on n'a plus rien à perdre.

"Young thing, teenage, adolescent, honors student, kid, underage nympha, little fourteen-year-old flesh, little girl, babydoll", et enfin girl... "girl, there's that word again». L'héroïne de Hard Candy incarne cette posture malgré le fait qu'elle est très jeune, un petit chaperon rouge travesti en petit chaperon rouge pour dissimuler le loup qu'elle porte en elle. Ce monstre qui s'oppose au monstre qui en est un mais ne veut pas l'admettre, le pédophile Jeff: «I'm not the monster you think. » On le sait: le pathos généralisé veut qu'on entende les drames intimes des criminels. Comme le dit Zizek, notre culture place le berceau de notre subjectivité dans notre intimité cachée alors que la vie publique n'est qu'apparence. Pourtant, c'est bien cette prétendue vie intime qui sert d'écran à la vie publique, à ce qu'on est en société: la «vérité » serait dans le personnage public, le faux dans l'histoire qu'on raconte sur soi pour noyer le poisson. Le récit qu'on fait de notre intimité cachée, de ce qu'on est vraiment, sert ainsi d'alibi, c'est-à-dire d'ailleurs par rapport à cette version de soi offerte à l'espace public ; cet autre soi qui, au fond, est un étranger.

De cette histoire intime, Haley Stark n'a rien à faire, elle n'y croit pas. Tout comme ses allusions à sa propre «folie» sonnent faux (il n'y a pas de raisons, conscientes ou inconscientes, psychanalysables, qui peuvent donner un sens à ses gestes), sa relation à Jeff est elle aussi dépourvue d'un intérêt pour le récit privé. Ainsi, l'histoire qu'il raconte au sujet de sa cousine et de sa tante et qu'il présente comme la source de son «mal» ne lui attire, de la part de la jeune fille, aucune sympathie. Si le public est amené à suivre le fil (ténu) de cette explication psychonarrative, Haley Stark, elle, $\mathrm{y}$ est insensible, refusant confession et excuses, tout comme Jeff n'a pas réussi à l'amadouer en usant de psychologie. Contrairement au petit chaperon rouge placé en position d'infériorité par les incessantes questions que pose le loup, elle qui écoute et qui, comme l'enfant qu'on endort en lui racontant une histoire, est ravie par les mots (Garat, 2004, p. 184-185), Haley Stark est au final celle qui mène le bal. Comme le petit chaperon rouge décrit par Garat, elle «brille par sa clarté d'élocution, sa volubilité confiante, son intrépidité et son aisance verbale » (Garat, 2004, p. 184). Mais contrairement à la victime qui se trouve dans une position «faussement égalitaire» puisque c'est le loup qui domine l'échange, Haley Stark détient le verbe: non seulement elle interrompt Jeff, le fait taire ${ }^{12}$ (et le bâillonne littéralement), mais elle détient le pouvoir de la réplique ${ }^{13}$, manipulant non seulement les mots, la phrase mais la théorie critique: "That's the whole nature versus nurture question, isn't? Was I born a cute vindictive little bitch, or did society help make me this way? I go back and forth on that.» Jeff le pédophile est un producteur d'images (il se dit photographe), mais elle est une productrice de mots, et c'est par le mot qu'elle l'emporte sur lui (comme Erica Bain qui redouble ses gestes de violence par sa chronique radiophonique). Dès leur rencontre, ce sont les livres, romans et ouvrages scientifiques, qui sont placés au centre des activités de la jeune fille; elle se décrit comme une "goon» qui a toujours le nez fourré dans un livre. Et à la fin du film, c'est par la menace des révélations qu'elle fera à Janelle, premier amour de Jeff, qu'elle le convainc de se suicider.
C'est d'ailleurs là la spécificité de ces deux vigilantes: elles passent à l'acte, mais ce passage à l'acte est une forme de sublimation. Elles sont plus grandes que nature, au final on ne sait pas qui elles sont ${ }^{14}$. Elles sont le loup et le petit chaperon rouge et Charles Perrault lui-même, l'auteur qui fait passer l'oral dans le champ de l'écrit comme il fait passer la petite fille du simple voir au véritable savoir (Méchoulan, 1992, p. 497), de l'inconscience à la vigilance qui doit l'empêcher de répéter la même erreur. Et le conte est véritablement mû par un principe de répétition (répétition du crime par le loup, répétition des expressions par l'écrivain ${ }^{15}$ ) et a pour objectif d'empêcher la répétition de l'erreur - c'est le sens de la morale. Ce même principe de répétition, le meurtre en série, est évidemment au centre de Hard Candy et The Brave One: les vigilantes répètent leur geste, elles ne lâchent pas le morceau, haussant toujours le volume. Mais elles ne le font pas que par le biais du crime; elles traduisent, ou rêvent de traduire le crime par le biais de l'art.

Si la répétition peut être symptôme, elle devient sublimation quand elle nous fait passer dans le monde de l'art. Erica Bain crypte ses chroniques, puis anime une ligne ouverte sur les ondes où le public est invité à commenter les actes de ce mystérieux vigilante qui n'est autre qu'elle. Haley Stark, pour sa part, envisage qu'on puisse faire un film:

- How hard do you think they'll look for me? The police find a pedophile killer all gift-wrapped for them, you really think they're gonna care who did their work for them? You think they're even gonna bring it up?

- Someone will. Some prosecutor'll make a career out of you.

- Uno problemo with that theory, Uncle Jeff. They never try girls as adults. Sexist but true. Plus I'll have the biggest legal defense fund you ever saw. If everyone who's ever been molested send in just five dollars, I'll be able to afford any lawyer I want. The worst, absolute worst case scenario? I do two years community service with psych evaluations, and Jodie Foster directs the movie version of the whole thing. Who do you want to play you?

Les enfants adorent les contes de fées, comme ils jouissent de frémir quand on leur raconte des histoires d'horreur, la sublimation «rend l'horreur vivable et pensable»(Garat, 2004, p. 215) ${ }^{16}$. Et cet effort de sublimation est ici manifeste. Néanmoins, reste qu'il s'agit d'attaques sur le réel, dans le réel, d'un exercice de la jus- 
tice qui n'en passe plus par les dispositifs sociaux. Entre le père-loi et le pire-loup, on choisit le pire et ça devient une autre loi.

Alors que le corps social est des plus dociles, souligne Agamben, le pouvoir s'inquiète: l'homme ordinaire serait le pire des terroristes. C'est un paradoxe apparent: "plus les dispositifs se font envahissants... plus le gouvernement se trouve face à un élément insaisissable qui semble d'autant plus se soustraire à sa prise qu'il s'y soumet avec docilité » (Agamben, 2007, p. 49). L'héroïne de The Brave One profane les dispositifs. D'aucuns ont dit que le personnage se matérialisait à partir du moment où elle possédait une arme. Je dirais plutôt qu'elle dématérialise cette arme, ce phallus, s'en sert à des fins non capitalistes, ne gagnant rien, et amenant ainsi à la lumière "cet Ingouvernable qui est tout à la fois le point d'origine et le point de fuite de toute politique » (Agamben, 2007, p. 50). Resterait donc cet ingouvernable, ce monstre, cet alien qui est «la vie ellemême».

Vivre ou mourir, ou peut-être être ou ne pas être, être sans que ce soit pour la dernière fois... voilà, pourrait-on dire, les enjeux qui se trament dans ces films, lutte entre un petit chaperon rouge et le «il était une fois » qui signe son entrée en littérature comme le début d'une souffrance à répétition. Toutes les petites filles, toutes les princesses de contes de fées sont (à un moment ou à un autre) des victimes. Le «il était une fois» est un mensonge, ou une parallaxe: selon où on se place, «il était une fois» signale la fois unique et un «il y en aura d'autres, infiniment, jusqu'à la fin des temps ». On enjoint les femmes à avoir confiance dans un système de justice - "We're the good guys» dit le policier à Erica Bain après son agression, qui lui répond: "How come it doesn't feel that way?" Mais ce que ces films pointent, c'est la posture refusée de victime et d'impuissante: les femmes toujours victimes réelles ou potentielles (pour reprendre les termes d'Elizabeth Badinter dans son essai Fausse route [2003]), les femmes victimes et donc témoins distants, désengagés. Ce qu'on nous donne, ici, c'est le rejet, aussi, du discours qui admet la victimisation des femmes dans le but de les faire taire.

Erica Bain et Haley Stark sont des bourreaux et elles ont raison de l'être, elles sont le résultat d'une logique monstrueuse moderne poussée à l'extrême, elles sont l'affirmation du "j'étais sur le lieu du crime au moment où il a été commis, nous sommes tous sur le lieu du crime, moi et vous aussi, le monstre est en nous ». La femme-monstre, la femme-loup est ce que devient le petit chaperon rouge, non pas parce qu'il n'a pas bien appris sa leçon mais parce que la leçon était un leurre, une façon de préparer la victime. Par les crimes qu'elles commettent, les femmes de Hard Candy et The Brave One expriment leur état de témoin moderne, de témoin toujours complice de la modernité et de ses dispositifs. Et comment survivre? Comment ne pas disparaître dans la mélancolie, la plainte ou le cynisme? Qu'est-ce qui reste de la femme, à l'heure du crime, à l'heure du loup? Reste peut-être la force $\mathrm{du}$ tremblement, car le pire n'est pas de trembler mais de toujours avoir peur.

\section{Bibliographie}

AGAMBEN, GIORGIO (2007). Qu'est-ce qu'un dispositif?, Paris, Payot et Rivage.

BADINTER, ÉLISABETH (2003). Fausse route, Paris, Odile Jacob.

FINKIELKRAUT, ALAIN et PETER SLOTERDIJK (2003). Les battements du monde, Paris, Fayard.

GARAT, ANNE-MARIE (2004). Une faim de loup, lecture du Petit chaperon rouge, Paris, Actes-Sud.

JORDAN, NEIL (2007). The Brave One, DVD-vidéo, son, coul., 122 min., Burbank, Warner Bros. Entertainement.

MÉCHOULAN, ÉRIC (1992). «Il n'y a pas de fées, il n'y a que des interprétations: lecture du Petit chaperon rouge», Papers on French Seventeenth Century Literature, vol. 19 , p. 489-500.

ORENSTEIN, CATHERINE (2003). Little Red Riding Hood Uncloaked, New York, Basic Books.

PERRAULT, CHARLES (2007). Le petit chaperon rouge, Paris, Gallimard.

SCORSESE, MARTIN (1999 [1976]). Taxi Driver, DVD-vidéo, son, coul., 135 min., Culver City, Columbia TriStar Home Video. SLADE, DAVID (2006). Hard Candy, DVDvidéo, son, coul., 105 min., Toronto, Maple. ZIZEK, SLAVOY (2006). The Parallax View, Cambridge, MIT Press.

\section{Notes}

1. L'un des exemples que donne Zizek est celui du vagin : incarnation du mystère de la sexualité, de ce qui est caché, et organe de la maternité, la naissance de l'enfant étant de l'ordre de la visibilité.

2. De jeunes adolescentes victimes de pédophiles dans le cas de Hard Candy; des jeunes femmes victimes de leur mari ou de leur proxénète; et, enfin, une petite fille victime du beau-père qui a sa garde après avoir assassiné sa mère dans The Brave One.

3. Produits en 2005 et en 2007, respectivement, et qui, avec d'autres films comme Death Sentence de James Wan (également en 2007), signalent un retour du "vigilante film» à Hollywood, retour que d'aucuns ont associé à l'Amérique post-9/11.

4. Et il faut se rappeler le rôle d'Ellen Page dans Juno de Jason Reitman (2007).
5. «Emboîtements multiples, poupée gigogne, on n'en finit pas d'ouvrir ce Loup, de voir ce qu'il a dans le ventre, car ce ventriloque est un champion de ventripotence [...] Cette histoire est épuisante, elle est dévorante de personnages, elle en avale autant qu'elle en produit. » (Garat, 2004, p. 162.)

6. Erica Bain: "Finding places I never knew existed. But am I finding them or are they finding me?»

7. Anne-Marie Garat compare l'acte irréaliste du loup à celui du tueur en série: «La litote escamote la cruauté, mais pas sa portée imaginaire. La scène se déroule à une telle vitesse qu'elle devient onirique, elle a l'étrangeté fantastique des visions. En cela elle rejoint paradoxalement une réalité abominable: les serial killers et autres tueurs fous, dans la brutalité et la vitesse d'exécution de leurs actes, allient la démence à la froide intelligence technicienne, la sauvagerie à la précision maniaque.» (Garat, 2004, p. 120.)

8. Dans Hard Candy, au lieu du chasseur qui tire la petite fille du ventre du loup, c'est la fille qui, figurativement, tire le loup du corps du chasseur en lui retirant ses testicules.

9. Et c'est là la description qu'Erica Bain donne, dans une de ses chroniques à la radio, de la ville de New York.

10. Il faut rappeler l'étymologie de alien: alienare, rendre étranger, rendre autre; dérivé d'alienus, autre, lui-même dérivé d'alias (alibi) : ailleurs.

11. Nighthawks est d'ailleurs le nom donné au café où se rejoignent Haley Stark et Jeff la première fois.

12.Jeff: «Don't-» Haley: «You can save yourself so much time by just dropping that word from your vocabulary. I'm gonna do what I want Jeff.»

13. "Your conversational skills are deteriorating as the day goes on, I must say. » (Haley à Jeff).

14. «Who are you? » demande Jeff à Haley Stark à la fin du film. Et The Brave One est entièrement construit sur l'enquête qui vise à trouver qui est le vigilante.

15. Comme le montre clairement Éric Méchoulan (1992).

16. «Il faut toutes les constructions de l'éducation et de la culture pour nous convaincre de renoncer à nous entre-dévorer. Pour savoir sublimer ces pulsions cannibales en baisers amoureux et en rituels érotiques; les transformer en sourires polis, ou les socialiser en actes de pouvoir. » (Garat, 2004, p. 176.)

17. Stephen Mulhall décrit ainsi l'alien: "The alien's form of life is (just, merely, simply) life, life as such: it is not so much a particular species as the essence of what it means to be a species, to be a creature, a natural being » (dans Zizek, 2006, p. 118). 